\title{
Anisotropic dynamics of magnetic colloidal cubes studied by x-ray photon correlation spectroscopy
}

\author{
Antara Pal $\odot,{ }^{1, *}$ Md. Arif Kamal $\odot,{ }^{1}$ Thomas Zinn, ${ }^{2}$ Jan K. G. Dhont $\odot, 3,4$ and Peter Schurtenberger $\circledast^{1,5}$ \\ ${ }^{1}$ Division of Physical Chemistry, Department of Chemistry, Lund University, SE-221 00 Lund, Sweden \\ ${ }^{2}$ ESRF-The European Synchrotron, 38043 Grenoble, France \\ ${ }^{3}$ IBI-4, Biomacromolecular Systems and Processes, Forschungszentrum Jülich GmbH, D-52428 Jülich, Germany \\ ${ }^{4}$ Department of Physics, Heinrich-Heine University Düsseldorf, D-40225 Düsseldorf, Germany \\ ${ }^{5}$ Lund Institute of Advanced Neutron and X-ray Science (LINXS), SE-223 70 Lund, Sweden
}

(Received 16 November 2020; revised 15 January 2021; accepted 17 February 2021; published 11 March 2021)

\begin{abstract}
Herein we present our results on the investigation of the influence of external magnetic fields on the anisotropic collective dynamics of core/shell colloidal cubes having a hematite core and silica shell. Owing to the hematite cores, these micrometer-sized particles possess permanent dipole moments, which are at an angle with respect to the long diagonal of the cubes. As a result, they self-assemble into chains, which subsequently sediment to form higher-order structures. Using multispeckle ultrasmall-angle x-ray photon correlation spectroscopy, the anisotropic dynamics within these structures at the nearest-neighbor length scale was probed. The relaxation of the intermediate scattering function follows a compressed exponential behavior along all the different directions with respect to the external field-parallel, perpendicular, and at an angle $\approx 45^{\circ}$ —indicating hyperdiffusive behavior. We believe that the inhomogeneous distribution of stress points originating from the interplay of external field-induced (both gravitational and magnetic) alignment of the chains are responsible for the anomalous dynamics. The effective diffusion coefficients along and at $\approx 45^{\circ}$ angle exhibit mild de Gennes narrowing, which is not very common for hyperdiffusive dynamics. We rationalize our observations by considering a superposition of diffusive and stress-induced ballistic processes and argue that depending on the azimuthal direction the relative contribution from these two processes changes.
\end{abstract}

DOI: 10.1103/PhysRevMaterials.5.035603

\section{INTRODUCTION}

Colloidal hard spheres have played a significant role in laying down the foundation of modern colloid science. However, with the emergence of a multitude of anisotropic, patchy, and/or responsive colloids the focus has gradually shifted from isotropic colloids to anisotropic ones. The self-assembly of anisotropic colloids has captured the imagination of the scientific community ever since the possibility to accomplish their self-assembly was demonstrated. Taking advantage of the shape anisotropy as well as the resulting anisotropy in the interaction potential of different anisotropic colloids, researchers have been able to manipulate their self-assembled structures [1-14]. Despite the enormous progress that has been achieved towards understanding the self-assembly of stimuli-responsive anisotropic colloids, a deeper understanding of their dynamical behavior is lacking.

In the case of magnetic colloidal suspensions, there is always a competition between the thermal and dipolar interac-

\footnotetext{
*antara.pal@fkem1.lu.se
}

Published by the American Physical Society under the terms of the Creative Commons Attribution 4.0 International license. Further distribution of this work must maintain attribution to the author(s) and the published article's title, journal citation, and DOI. Funded by Bibsam. tions. The particles stay well dispersed as long as the thermal energy wins over the dipolar interaction. However, in the presence of an external field, when the dipolar interaction becomes strong enough to overcome the thermal energy, they start to form chainlike aggregates. There is a scarcity of reports in the literature on the mesoscopic dynamics at the nearest-neighbor length scale for these systems undergoing chain formation. One of the primary reasons behind this scarcity of knowledge can be attributed to the fact that the scattering-based traditional techniques like dynamic light scattering (DLS) (used to measure the dynamics of colloidal particles) are limited by the opacity of the samples. Microscopy-based techniques like particle tracking, on the other hand, are always limited in quasi-two-dimensions. Thanks to the recent development of two alternative techniques, namely, differential dynamics microscopy and multispeckle x-ray photon correlation spectroscopy (XPCS), it is now possible to measure the dynamics of turbid samples in three dimensions [5,15].

In recent times, synchrotron-based multispeckle XPCS using coherent $\mathrm{x}$ rays has emerged as an alternative technique to study dynamics in diverse soft matter systems such as colloids [16-18], polymers [19], gels [20-22], and supercooled liquids $[23,24]$. Although the basic principle of XPCS is analogous to that of DLS, the use of $x$ rays instead of visible light allows one to circumvent the problems related to multiple scattering and absorption that are often encountered in DLS when opaque systems like magnetic colloids are investigated. Most of the experimental studies reported in the literature that have 
used XPCS to study the dynamics of magnetic colloids have predominantly been restricted to the use of spherical particles $[18,25-30]$. It has been reported that spherical particles that self-assemble into chains under the influence of an external field exhibit anisotropic dynamics [30]. The presence of anisotropy in the particle shape as well as in their interaction potential would give rise to complexity in the self-assembled structures and their resulting dynamics. When the particle shape is inherently anisotropic, the external field profoundly influences the overall interparticle interaction potential. The field induces an additional magnetic dipolar contribution. The combined anisotropy in orientation and interactions results in the suspension dynamics to become anisotropic, with a distinctly different behavior perpendicular and parallel to the direction of the applied field. Only a handful of studies exist in the literature that reports the dynamics of anisotropic magnetic particles $[15,16,31,32]$.

In this paper, we report the translational dynamics of colloidal cubes under the influence of an external magnetic field. Using XPCS in the ultrasmall angle regime we have investigated the collective dynamics of field-induced particle assemblies on length scales from mesoscopic dimensions of several particle diameters to distances below the nearest-neighbor distance, i.e., smaller than the singleparticle dimension. Being made up of hematite cores and silica shells, these particles self-assemble into chains, which sediment and self-assemble into higher-order gel-like structures. Consequently, both the resulting scattering patterns and the associated dynamics are highly anisotropic. Diffusion was found to be anomalous in directions both parallel and perpendicular to the field direction. In addition, it was also found to be anomalous at an angle $\approx 45^{\circ}$ (diagonal) to the direction of the field. The correlation functions can be described by a compressed Kohlrausch-Williams-Watts (KWW) exponential function. With increasing field strength, structural correlation builds up in a direction parallel and diagonal to the field direction. Surprisingly, the effective diffusion coefficients along these directions exhibit a mild de Gennes narrowing. In contrast, we observe neither the structural correlation nor the de Gennes narrowing in a direction perpendicular to the field direction.

\section{EXPERIMENTAL SECTION}

\section{A. Synthesis}

Micrometer-sized hematite cubes were prepared following the gel-sol method proposed by Sugimoto and Sakata [33]. In a typical synthesis, $20 \mathrm{~g} \mathrm{NaOH}$ dissolved in $100 \mathrm{~mL}$ water was quickly added to $50 \mathrm{~g} \mathrm{FeCl} 3 \cdot 6 \mathrm{H}_{2} \mathrm{O}$ (Sigma-Aldrich, 99\%) dissolved in $100 \mathrm{~mL}$ water under vigorous magnetic stirring. Subsequently, the obtained condensed iron hydroxide gel was aged at $10^{\circ} \mathrm{C}$ for 8 days. The resulting precipitate of the particles was then purified by repeated centrifugation and redispersion cycles in water.

In the next step, the hematite particles were coated with an additional silica layer by following Graf. et al. [34]. As an initial step, the particles were coated with a sterically stabilizing layer by adding an excess of polyvinylpyrrolidone (PVP) to an aqueous hematite particle dispersion while stirring. The dispersion was stirred overnight and was finally washed with
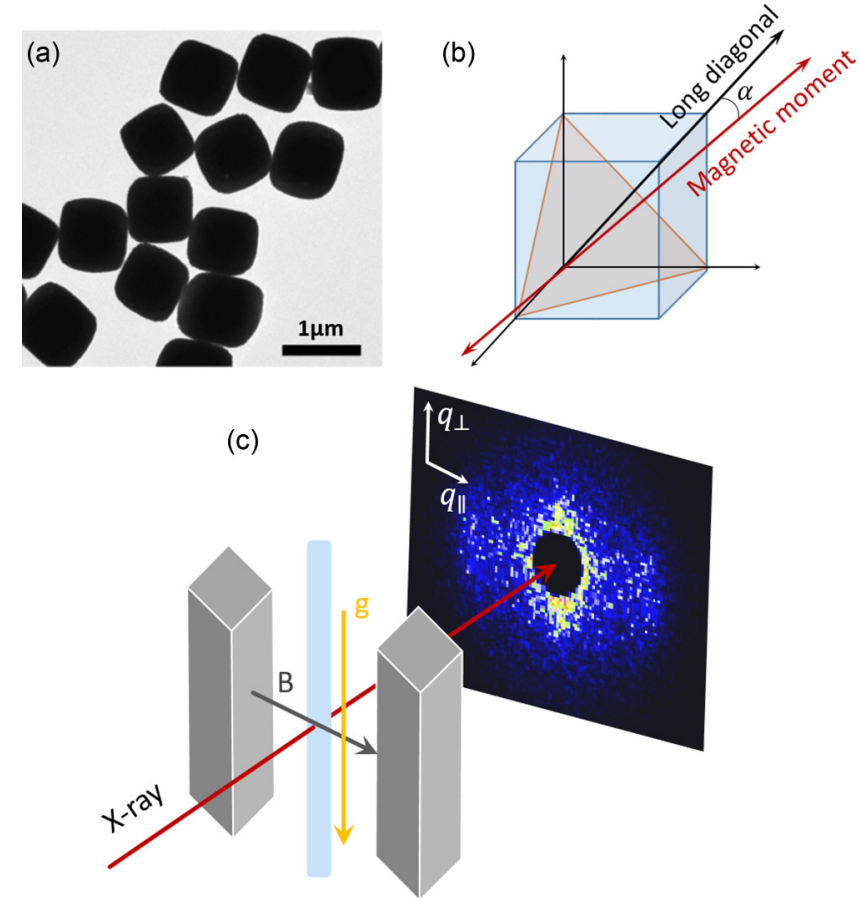

FIG. 1. (a) A representative TEM image of core-shell hematitesilica cubic colloids. (b) A schematic showing the direction of the magnetic moment (red), which is oriented at an angle $\alpha$ with respect to the long diagonal ([111] direction) of the cube. The orange triangle indicates the [111] plane. (c) Experimental scheme for the multispeckle ultrasmall-angle $\mathrm{x}$-ray photon correlation spectroscopy experiment. In this case, the $\mathrm{x}$-ray beam, external magnetic field (B) direction, and the direction of gravity $(\mathrm{g})$ are perpendicular to each other.

ethanol. The silica coating was performed in a 3-1 round bottom flask under mechanical stirring and ultrasonication. A mixture of 11 ethanol, $100 \mathrm{~mL}$ water, and $15 \mathrm{~mL}$ of 1 wt $\%$ tetramethylammonium hydroxide (TMAH) solution was prepared and $75 \mathrm{~mL}$ of $5 \mathrm{wt} \%$ PVP-stabilized hematite dispersion in ethanol was added. Subsequently, using a peristaltic pump tetraethyl orthosilicate (TEOS) solution was added to the mixture at the rate of $16 \mathrm{~mL} / \mathrm{h}$. The TEOS solution was prepared by mixing $10 \mathrm{~mL}$ of TEOS and $10 \mathrm{~mL}$ of ethanol. To prevent aggregation, a solution of $20 \mathrm{~g}$ PVP in $100 \mathrm{~mL}$ water was added to the dispersion, which was then sonicated for 2 more hours and stirred overnight. The silica-coated particle dispersion was washed several times with ethanol and then with water. Finally, the particles were redispersed in a 50-50 glycerol-water (wt-wt) mixture in order to prevent fast sedimentation. It has been shown before that a water-glycerol mixture can be used to slow down the dynamics in order to match the speed of the two-dimensional (2D) detector without any beam damage while measuring XPCS [35]. The final concentration of the particles was $\sim 1 \mathrm{wt} \%$.

\section{B. Characterization and methods}

The cube-shaped colloidal particles were characterized using transmission electron microscopy (TEM) (JEM-3000F) [Fig. 1(a)]. By analyzing more than 100 particles in the TEM 
images, the average edge length of these particles was found to be $935 \pm 50 \mathrm{~nm}$. Since the dimension of the hematite core of these particles is large, they possess permanent magnetic dipole moments. The direction of the dipole moment makes an angle $\alpha=12^{\circ}$ with respect to the long diagonal ([111] direction) as shown in Fig. 1(b) [36].

In view of the large size and high opacity of the particles, we chose multispeckle ultrasmall-angle $\mathrm{x}$-ray photon correlation spectroscopy to probe their dynamics $[37,38]$. Experiments were performed at the beamline ID02, ESRF, in a pinhole ultrasmall-angle $\mathrm{x}$-ray scattering geometry. This unique instrument allows XPCS measurements on particle suspensions down to the $\mu \mathrm{m}^{-1} q$ range. A schematic of the experimental setup is shown in Fig. 1(c). The dispersion was filled in 1-mm capillaries, which were then flame sealed in order to prevent evaporation of water. In the next step, the individual capillary was placed vertically between two poles of a permanent magnet as shown in Fig. 1(c). The strength of the magnetic field at the sample position was then adjusted by widening the gap between the poles. At the largest distance, the field is of the order of the earth magnetic field, and we will refer to this case as zero field $B=0$. Due to the large size of the hematite cores, these cubes self-assemble into dipolar chains at the Earth's magnetic field or the residual field from the permanent magnet at the zero field position. As a result, they sediment quite fast even when a water-glycerol mixture was used as the solvent. The static and dynamic measurements were performed at the bottom of the capillaries on the sedimented sample at $25^{\circ} \mathrm{C}$ using an $\mathrm{x}$ ray of wavelength $0.995 \AA$ and a sample to detector distance of $30.7 \mathrm{~m}$. The two-dimensional $\mathrm{X}$-ray detection was achieved using an Eiger $500 \mathrm{~K}$ detector. The correlation functions were calculated using the PyXPCS software package, developed at the beamline ID10, ESRF. To obtain the direction-dependent correlation functions, calculations were performed within three azimuthal sectors, each defining a triangular region of interest along, perpendicular, and at an angle of approximately $45^{\circ}$ to the field directions with an azimuthal width of approximately $45^{\circ}$.

\section{RESULTS AND DISCUSSION}

Both DLS and XPCS provide information about particle dynamics via a measurement of the intensity-intensity autocorrelation function,

$$
g^{(2)}(\vec{q}, t)=\frac{\langle I(\vec{q}, \tau) I(\vec{q}, \tau+t)\rangle}{\langle I(\vec{q})\rangle^{2}},
$$

where $\vec{q}$ is the scattering vector with magnitude $q=$ $4 \pi \sin (\theta / 2) / \lambda, \theta$ being the scattering angle. In both cases, $g^{(2)}(\vec{q}, t)$ is related to the decay of spontaneously created density fluctuations of wavelength $2 \pi / q$. Particle dynamics characterized by XPCS correspond to collective motion, and the explicit $q$ and time dependence of the measured correlation functions depends on the relevant mechanisms responsible for particle motion on these length and time scales. Given the field-induced self-assembly of individual particles into chains and their alignment, we do expect a highly anisotropic structure and dynamics, and thus need to characterize these properties accordingly along different principal directions in the reciprocal space. Figure 2 represents the structure and dynamics parallel to the direction of the external field. In the static scattering profile along the direction of the field [Fig. 2(a)], one can observe that at all field values there is a well-defined peak at $q=0.0062 \mathrm{~nm}^{-1}$, which is in reasonable agreement with the edge length $(d)$ of the cubes and likely the result of a structure factor peak that is related to strong positional correlations. The presence of a peak in the scattered intensity profile at zero magnetic field at such low concentrations ( $1 \mathrm{wt} \%)$ indicates that due to their large dipole moments, the particles self-assemble into higher-order structures like dipolar chains at the Earth's magnetic field or at the residual magnetism of the permanent magnet that was used. The self-assembled structure becomes increasingly prominent with increasing field strength as reflected by the increase in the height of the structural correlation peak as a function of field strength. The dashed lines in Fig. 2(a) represent the $q$ values at which we have measured the dynamics of the system. Figure 2(b) represents the intensity autocorrelation functions, $g_{\|}^{(2)}(q, t)$, for different $q$ values. The intermediate scattering function $g^{(1)}(\vec{q}, t)$ is related to $g^{(2)}(\vec{q}, t)$ via the Siegert relation, $g^{(1)}(\vec{q}, t)=\sqrt{\left[g^{(2)}(\vec{q}, t)-1\right] / \beta}$, where $\beta$ is the coherence factor of the experimental setup. Along the direction of the field, $g_{\|}^{(1)}(q, t)$ can be described phenomenologically by a KWW expression,

$$
g_{\|}^{(1)}(q, t)=\exp \left[-\left(t / t_{c \|}\right)^{\gamma}\right]
$$

where $t_{c \|}$ is the relaxation time and $\gamma$ is the KWW exponent that depends on the mechanism responsible for the particle motion. The mean value of $t_{c \|}$ is $\left\langle t_{c \|}\right\rangle=\left(t_{c \|} / \gamma\right) \Gamma(1 / \gamma)$, where $\Gamma$ is the Euler gamma function [39]. For a generalized KWW model, $g^{1}(q, t)$ can be considered as arising from a superposition of exponentials, which has a distribution function $\rho_{w w}$ such that

$$
\exp \left(-\left[t / t_{c}\right]^{\gamma}\right)=\int_{0}^{\infty} \exp (-t / \tau) \rho_{w w}(\tau) d \tau .
$$

The gamma function correction takes this distribution function into account, and $\left\langle t_{c}\right\rangle$ thus corresponds to an effective or mean relaxation time.

For freely diffusing (Brownian motion) particles $\gamma=1$ and $\left\langle t_{c \|}\right\rangle=t_{c \|}$. In this case the relaxation time is related to the collective diffusion constant $D$ by $1 /\left\langle t_{c \|}\right\rangle=D q^{2}$. However, for most of our correlation functions, we found $\gamma>1$, which corresponds to a compressed exponential behavior that may signify a hyperdiffusive process. Such a hyperdiffusive relaxation has, for example, been observed in colloidal gels, where one finds $\gamma \approx 1.5$ and $1 /\left\langle t_{c \|}\right\rangle \propto q[40,41]$. Given the different characteristic $q$ dependence observed for various relaxation scenarios in colloidal fluids and solids, we have tried to fit the relaxation rates $1 /\left\langle t_{c_{\|}}\right\rangle$shown in Fig. 2(c) with a power-law behavior, $1 /\left\langle t_{c \|}\right\rangle \propto q^{n_{\|}}$(continuous lines). We find a clear variation of the exponent $n_{\|}$as a function of $\mathrm{B}$ as shown in Fig. 2(d). Moreover, while the overall $q$ dependence of $1 /\left\langle t_{c_{\|}}\right\rangle$ is reasonably well described by a power law, one can observe some systematic deviations of the experimental data around the fitted lines (especially for $100 \mathrm{mT}$ ) around the location of the peak in the static scattering intensity [Fig. 2(a)], reminiscent of the relationship between the structure factor and the $q$-dependent collective diffusion coefficient for strongly interacting colloidal particles [42]. 

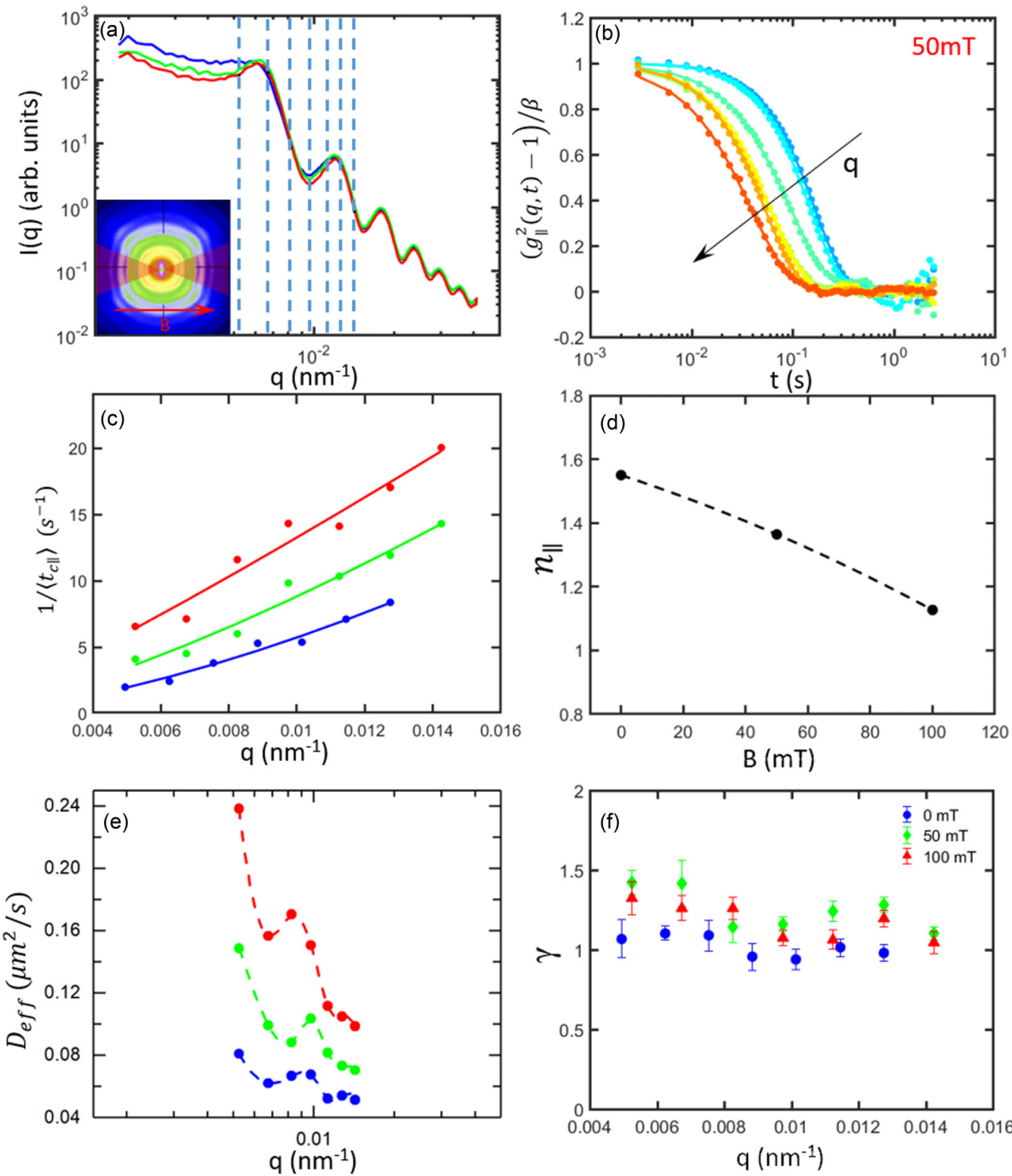

FIG. 2. Dynamics at an angle $0^{\circ}$ (parallel) with respect to the applied field. (a) Variation of scattered intensity $I$ as a function of scattering vector $q$ at different magnetic fields. The vertical dashed lines represent the $q$ values at which the dynamics was measured. The inset shows a representative $2 \mathrm{D}$ diffraction pattern where the magnetic field $(\mathrm{B})$ is along the horizontal direction at a field value of $50 \mathrm{mT}$. An azimuthal sector average has been taken in the shaded region to study the dynamics along the direction of the field. (b) Intensity autocorrelation functions for different $q$ values indicated by the dashed lines in (a). The symbols represent the experimental data while the lines correspond to the fit with the KWW model. (c) Observed variation of the relaxation rate $1 /\left\langle t_{c \|}\right\rangle$ as a function of $q$ (symbols), which is fitted with the expression $1 /\left\langle t_{c \|}\right\rangle \propto q^{n_{\|}}$(continuous lines). (d) The variation of the exponent $n_{\|}$as a function of B. (e) Variation of the effective diffusion coefficients as a function of $q$ for different field values. The lines are a guide to the eye. (f) Variation of the compressed exponent, $\gamma$, as a function of $q$. Identical color scheme has been used for (a), (c), and (d). Error bars are smaller than the symbol size for (c) and (e).

We have tried to further investigate this point by defining an effective diffusion coefficient, $D_{\text {eff }}=1 /\left(\left\langle t_{c} \|\right\rangle q^{2}\right)$ shown in Fig. 2(e) as a function of $q$; different colors correspond to different field values. For all the field values measured, $1 / D_{\text {eff }}$ follows a similar trend as a function of $q$ as the scattering intensity (I) [Fig. 2(a)]. This particular behavior is known as de Gennes narrowing, which for short-time collective diffusion can be mathematically expressed as $D_{\text {eff }} / D_{0}=$ $H(q) / S(q)$ where $D_{0}$ is the free particle diffusion coefficient, $H(q)$ is the hydrodynamic function, and $S(q) \propto I(q) / P(Q)$ is the structure factor [42]. The origin of de Gennes narrowing can be attributed to the spatial correlations between the nearest-neighboring particles and is an indication of the sensitivity of the collective relaxation processes with respect to the structure. XPCS (or DLS) measures the decay of density or concentration fluctuations on length scales of $1 / q$ $[42,43]$. In fluidlike systems, this occurs via collective diffusion, which in turn depends on the volume-fraction-dependent time-averaged structural correlations between particles on these characteristic length scales. At the peak of the struc- 

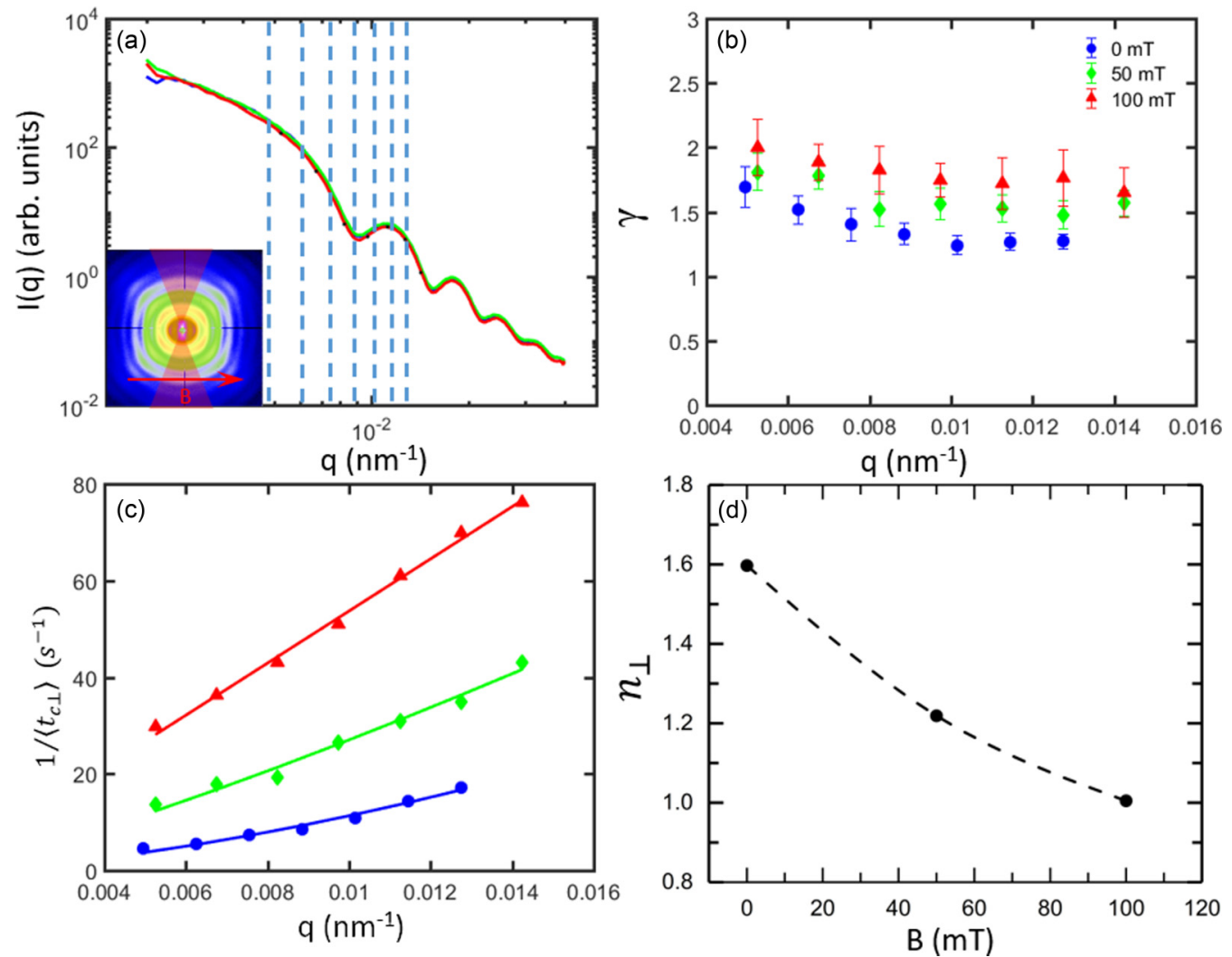

FIG. 3. Dynamics at an angle $90^{\circ}$ (perpendicular) with respect to the applied field. (a) Variation of scattered intensity $I$ as a function of scattering vector $q$ at different magnetic fields. The vertical dashed lines represent the $q$ values at which the dynamics was measured. The inset shows a representative 2D diffraction pattern where the magnetic field (B) is along the horizontal direction at a field value of $50 \mathrm{mT}$. An azimuthal sector average has been taken in the shaded region to study the dynamics along the direction of the field. (b) Variation of the compressed exponent, $\gamma$, as a function of $q$. (c) Observed variation of the relaxation rate $1 /\left\langle t_{c \perp}\right\rangle$ as a function of $q$ (symbols), which is fitted with the expression $1 /\left\langle t_{c \perp}\right\rangle \propto q^{n_{\perp}}$ (continuous lines). (d) The variation of the exponent $n_{\perp}$ as a function of B. The continuous line is a guide to the eye. Identical color scheme has been used for (a), (b), and (c). Error bars are smaller than the symbol size for (c) and (e).

ture factor, i.e., at the nearest-neighbor distance, collective diffusion is a measure of the dynamical relaxation of the nearest-neighbor cage structure, which slows down with increasing structural correlations (or peak height), as expressed by the de Gennes narrowing. While de Gennes narrowing is widely observed for diffusive motion $[43,44]$, its occurrence for hyperdiffusive motion is rare. For all the field values, the "compressing exponent," $\gamma$, lies approximately between 1 and 1.5 [Fig. 2(f)]. A similar trend has been reported earlier for different systems with analogous dynamics $[18,23,40,45]$. However, for most of these cases $\gamma \approx 1.5$ and $1 /\left\langle t_{c \|}\right\rangle \sim q$, which we do not observe in our case; instead we observe a mild de Gennes narrowing.

Figure 3 represents the structural and dynamical properties observed in a direction perpendicular to the direction of the external field. The intensity profiles [Fig. 3(a)] show no detectable structural correlations along this direction. Similar to the parallel direction, $g_{\perp}^{(1)}(q, t)$ can also be described by the generalized KWW model. Figure 3(b) exhibits the variation of the compressing exponent, $\gamma$, as a function of $q$, which shows that the value of $\gamma$ along this direction lies between 1.25 and 2. However, in agreement with the missing structural features in the intensity profiles, we do not observe any de Gennes narrowing in this direction. Rather, the $\left\langle t_{c \perp}\right\rangle$ data appear to follow a power-law behavior with $1 /\left\langle t_{c \perp}\right\rangle \propto q^{n_{\perp}}$ [Fig. 3(c)], where $n_{\perp}$ is varying as a function of the external magnetic field; starting from a higher value of 1.6 at the lowest field it saturates at 1 at the highest field [Fig. 3(d)].

Figure 4 demonstrates the structure and dynamics along $\approx 45^{\circ}$ (diagonal) with respect to the external field. From Fig. 4(a) it is evident that even at the zero field there are visible structural correlations, similar to those observed along the direction of the field as shown in Fig. 2. Further, one can fit the correlation functions with the KWW model to get the correlation time (which is related to the effective diffusion coefficient) and the KWW exponent $\gamma$ [Fig. 4(b)]. Surprisingly, in this case, the dynamics is also hyperdiffusive. Similar to what has been observed along the parallel direction, we again find characteristic deviations of $1 /\left\langle t_{c_{D}}\right\rangle$ from a simple power law, $1 /\left\langle t_{c_{D}}\right\rangle \propto q^{n_{D}}$ [Figs. 4(c) and 4(d)], which become more pronounced with increasing field strength. When plotting the effective diffusion coefficient $D_{\text {eff }}(q)$ instead, we again observe a weak de Gennes narrowing [Fig. 4(e)]. The compressing exponent $\gamma$ varies between 1.0 and 1.5; the same as that along the direction of the field [Fig. 4(f)].

The results summarized in Figs. 2-4 appear contradictory and inconsistent when looking at them with our initial expectations in mind. We expect the hematite cubes to align and form chains in the presence of an external magnetic field, and the chain size to increase with increasing field strength. 

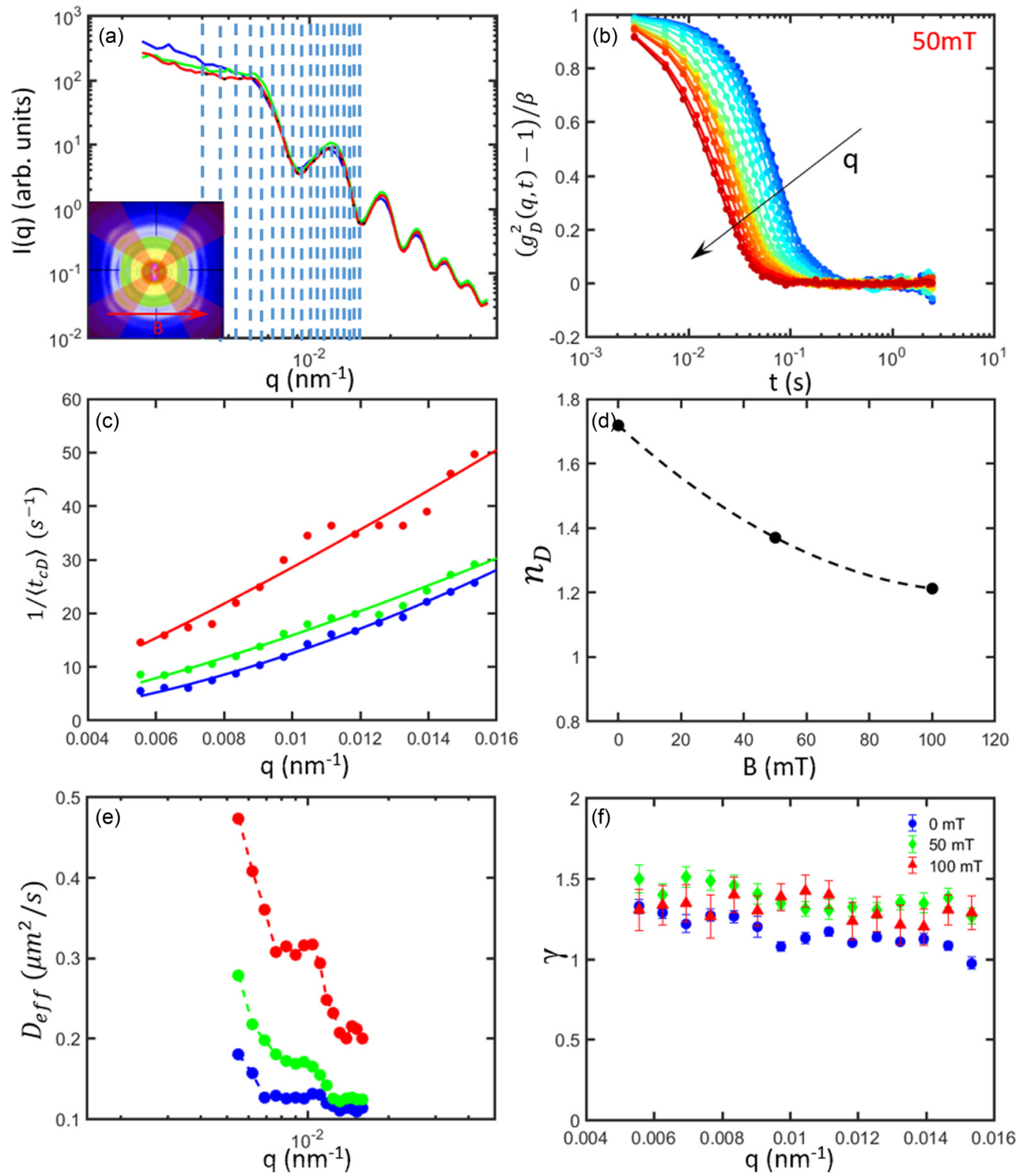

FIG. 4. Dynamics at an angle $\approx 45^{\circ}$ (diagonal) with respect to the applied field. (a) Variation of scattered intensity $I$ as a function of scattering vector $q$ at different magnetic fields. The vertical dashed lines represent the $q$ values at which the dynamics was measured. The inset shows a representative 2D diffraction pattern where the magnetic field (B) is along the horizontal direction at a field value of $50 \mathrm{mT}$. An azimuthal sector average has been taken in the shaded region to study the dynamics at an angle $\approx 45^{\circ}$ with respect to the field. (b) Intensity autocorrelation functions for different $q$ values indicated by the dashed lines in (a). The symbols represent the experimental data while the lines correspond to the fit with KWW model. (c) Observed variation of the relaxation rate $1 /\left\langle t_{c_{D}}\right\rangle$ as a function of $q$ (symbols), which is fitted with the expression $1 /\left\langle t_{c_{D}}\right\rangle \propto q^{n_{D}}$ (continuous lines). (d) The variation of the exponent $n_{D}$ as a function of B. (e) Variation of the effective diffusion coefficients as a function of $q$ for different field values. The lines are a guide to the eye. (f) Variation of the compressed exponent, $\gamma$, as a function of $q$. Identical color scheme has been used for (a), (c), and (d). Error bars are smaller than the symbol size for (c) and (e).

Moreover, it has already been demonstrated that the dipole moment of the individual hematite cubes lies in a particular direction with respect to the long diagonal [36]. Therefore, the chains will initially form at an angle with respect to the field. Chain formation should result in well-defined structural order along the chain axis, and therefore in a pronounced structure factor with a peak at $q_{\max } \approx 2 \pi / r_{d}$, where $r_{d}$ is the length of the long diagonal of a cube, along a direction of approximately $45^{\circ}$ with respect to the field direction. Similar but weaker structural correlations should then appear parallel and perpen- dicular to the field, where we would see the corresponding structural component of $S(q)$ projected onto these two directions. However, this is not what we observe. Figures 2(a)-4(a) show instead a pronounced peak in the intensity profile at $q_{\max } \approx 2 \pi / d$ parallel to the field, a weaker peak with a similar value of $q_{\max }$ at $45^{\circ}$, and no detectable structural correlations perpendicular to the field. We can understand this finding by taking into account the size and density of the particles. As the chains gradually grow in size, they start sedimenting under the influence of gravity, and gradually self-assemble 


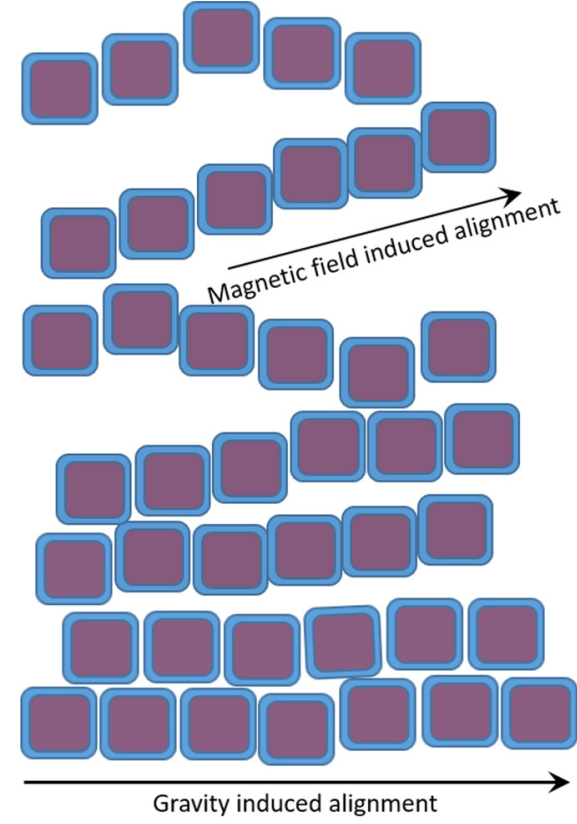

B

FIG. 5. Interplay between the external magnetic force, which is trying to align the chains at an angle, and the gravitational force, which is trying to minimize the gravitational energy by aligning them parallel to the external magnetic field.

into a three-dimensional structure at the bottom of the sample. Gravitational compression then tries to align these sedimented chains parallel to the external field direction as illustrated in Fig. 5, and we thus expect to find preferential chain alignment along the field direction in the bottom of the sedimented sample where our experiments were conducted. However, the combination of the external field that tries to maintain the most favorable chain alignment angle, the expected broad chain length distribution, excluded volume chain-chain interactions, and thermal motion will result in a three-dimensional network of chains with preferred but incomplete alignment along the field direction, but no well-defined stacking order of the particle chains perpendicular to the field. As a result, one would expect to observe structural correlations primarily in the form of vertical lines perpendicular to the direction in which the chains align as seen in the diffraction pattern [Fig. 2(a) inset]. Peaks in the intensity profiles are indeed observed along and at $\approx 45^{\circ}$ angle with respect to the external field [Figs. 2(a) and 4(a), respectively] but not perpendicular to it [Fig. 3(a)].

This structural order also must have important consequences for the dynamics on the relevant length scales measured by XPCS. For dense suspensions of strongly correlated particles, where interparticle interactions result in a $q$-dependent structure factor $S(q)$, we expect the decay of $g^{(2)}$ to strongly depend on the magnitude of $q$ relative to the position $q_{\max }$ of the nearest-neighbor peak in the structure factor $S(q)$. We can understand this by considering that the relaxation of these density fluctuations will be linked to collective particle motion on the corresponding length scale, and we expect that the underlying mechanisms responsible for these collective dynamics will be strongly influenced by the degree of correlation seen on these length scales. For $q \ll q_{\max }$, XPCS thus probes fluctuations whose wavelength is much larger than the interparticle spacing, which is characterized by the so-called gradient diffusion coefficient. Density fluctuations on length scales of the nearest-neighbor distance, on the other hand, are dominated by structural relaxation and for $q \gg q_{\max }$ by small-scale motions of individual particles within the cage of nearest neighbors. As described above, for particles undergoing Brownian motion, the $q$-dependent diffusion coefficient will follow the inverse of the structure factor, i.e., $D_{\text {eff }}(q) \sim 1 / S(q)$. While we indeed see such a qualitative behavior in the directions parallel and at an angle of $45^{\circ}$ with respect to the external field, where we see measurable structural correlations [Figs. 2(e) and 4(e)], the measured dynamic properties are much more complex. Most importantly, we do not see the typical exponential decay characteristic for Brownian motion, or the subdiffusive behavior frequently observed for hard-sphere particles in the vicinity of an arrest transition or colloidal gels formed through irreversible aggregation of individual particles. Instead, the correlation functions showed a compressed exponential decay indicating so-called hyperdiffusive dynamics.

The dynamics observed perpendicular to the field are summarized in Fig. 3. Here we find a compressed exponential decay of $g^{(2)}(q, t)$ with $\gamma \approx 1.5$, and a $q$ dependence of relaxation times that follows a power law with a field-dependent exponent that decreases from $n \approx 1.6$ at zero fields to 1 at maximum field strength. There are interesting and close similarities with the behavior found for colloidal gels, which also exhibit hyperdiffusive behavior $[41,46]$. There a mechanism has been postulated where the gels locally collapse to form small dense clusters, so-called "microcollapses." Such a microcollapse leads to a deformation of the remaining part of the gel, and thus induces long-ranged stresses. The accompanying gradients in stresses in turn lead to temporary ballistic motion of the remaining particles towards the collapsed region. It has been shown in Refs. [43,44] that the contribution from such ballistic motion leads to a hyperdiffusive exponent of 1.5 and a linear $q$ dependence. In our case, the stress arises not due to local microcollapses within the network structure caused by ongoing aggregation processes but results from the interplay between the external field-induced alignment of the chains and the gravitational compression arising due to sedimentation. Owing to the presence of these two competing forces, a long-ranged stress field develops in the sedimented structures. Relaxation of this stress field, which results from the rearrangement of the individual particles and chains, is responsible for the compressed exponential behavior observed.

This is also supported by the fact that the resulting relaxation times shown in Fig. 3(c) decrease with increasing field strength. If these relaxation processes characterized by XPCS were due to the typical thermal motion of individual particles and clusters, we would expect the thermal motion to slow down and the corresponding relaxation times to increase with external field strength due to the fact that the average chain length will increase. Further, the typical diffusion times over a nearest-neighbor distance corresponding to the particle diameter (or $2 \pi / q_{\max }$ ) for these large colloidal cubes can be estimated as $\left(2 \pi / q_{\max }\right)^{2} / D_{\text {eff }}\left(q_{\max }\right)$. The measured relaxation 
TABLE I. Comparison of the effective diffusion coefficients and $\gamma$ between different azimuthal directions.

\begin{tabular}{|c|c|c|c|c|c|c|}
\hline \multirow[b]{2}{*}{$B$} & \multicolumn{2}{|c|}{ Parallel } & \multicolumn{2}{|c|}{ Diagonal } & \multicolumn{2}{|l|}{ Perpendicular } \\
\hline & $D_{\text {eff }}\left(q_{\max }\right) / D_{0}$ & $\gamma$ & $D_{\text {eff }}\left(q_{\text {max }}\right) / D_{0}$ & $\gamma$ & $D_{\text {eff }}\left(q=0.00675 \mathrm{~nm}^{-1}\right) / D_{0}$ & $\gamma$ \\
\hline $0 \mathrm{mT}$ & $0.82 \pm 0.01$ & $1.1 \pm 0.04$ & $1.66 \pm 0.01$ & $1.3 \pm 0.04$ & $1.8 \pm 0.01$ & $1.5 \pm 0.11$ \\
\hline $50 \mathrm{mT}$ & $1.16 \pm 0.01$ & $1.1 \pm 0.1$ & $2.27 \pm 0.01$ & $1.5 \pm 0.06$ & $5.16 \pm 0.01$ & $1.8 \pm 0.11$ \\
\hline $100 \mathrm{mT}$ & $2.07 \pm 0.01$ & $1.3 \pm 0.08$ & $4.14 \pm 0.01$ & $1.4 \pm 0.11$ & $12.22 \pm 0.01$ & $1.9 \pm 0.14$ \\
\hline
\end{tabular}

times in Fig. 3(c) are 2-12 times smaller than that of free diffusion (Table I) indicating that along this direction the major contribution is from a ballistic process.

Another mechanism that could be responsible for the observed decay in $g^{(2)}(q, t)$ could arise from thermally induced breaking of bonds in the field-induced assembly of particle chains. We can estimate the lifetime of temporary bonds in the chains (or particle escape time from the attractive well) created by the attractive interaction between the particles in a chain. An estimate of the escape time from the attractive well using Kramers theory, namely, $\tau_{b} \approx\left(\Delta^{2} / D_{0}\right) \exp \left(-\epsilon / k_{B} T\right)$, where $\Delta$ is the width of the potential, $D_{0}$ the free diffusion coefficient of an individual cube $\left(0.08 \mu \mathrm{m}^{2} / \mathrm{s}\right)$, and $\epsilon$ the potential energy caused by the dipole-dipole interaction at contact, leads to relaxation times of order $10^{5} \mathrm{~s}$. In these calculations we have assumed magnetic properties (magnetic moment $/$ mass $=0.38 \mathrm{~J} / \mathrm{T} / \mathrm{kg})$ and density $\left(\rho=5300 \mathrm{~kg} / \mathrm{m}^{3}\right)$ of bulk hematite, which results in a magnetic moment for each cube $\mu=1.4682 e^{-15} \mathrm{~J} / \mathrm{T}$. Considering the expression for the contact potential to be $\epsilon=\mu_{0} \frac{\mu^{2}}{4 \pi r_{d}^{3}}\left(r_{d}=\sqrt{3} d\right)$, we found it to be $14 k_{B} T$. We have assumed a well width corresponding to $10 \%$ of the particle edge length. This high value of estimated relaxation time clearly indicates that density fluctuations cannot decay through thermal motion or spontaneous breakup of the field-induced chains on the time scales measured by XPCS, but favors a mechanism based on local stresses as discussed above.

An explanation for the observed de Gennes narrowing parallel and at an angle of approximately $45^{\circ}$ with respect to the field is not so straightforward. Let us first have a look at the variation of the KWW exponent $\gamma$ that describes deviations from an exponential decay of $g^{(1)}(q, t)$ as a function of $q$ and field strength for the different directions. It is seen that as the field strength increases from 0 to $100 \mathrm{mT}, \gamma$ varies from 1.1 to 1.4 along the parallel [Fig. 2(f)] and from 1.2 to 1.5 along the diagonal [Fig. 4(f)] direction, respectively. When a process is diffusive the value of $\gamma$ is 1 while a value higher than 1 indicates a ballistic process. Further as the field strength increases from 0 to $100 \mathrm{mT}$, the value of $D_{\text {eff }}\left(q_{\max }\right) / D_{0}$ increases from 0.8 to 2.1 along the parallel and from 1.7 to 4.1 along the diagonal direction, respectively (Table I). At higher concentrations when the particles are undergoing (restricted) Brownian motion the short-time cage diffusion coefficient $D_{\text {eff }}^{s}\left(q_{\max }\right) / D_{0}$ should decrease with increasing volume fraction [42]. However, the fact that we have $D_{\text {eff }}\left(q_{\max }\right) / D_{0} \approx 0.8$ at zero field for the direction parallel to the field combined with $\gamma \approx 1$ indicates that under these conditions the diffusion of individual particles might indeed contribute considerably to the observed decay of the correlation function on these length scales, resulting in the observation of a de Gennes narrowing. At higher fields, along this direction, $D_{\text {eff }}\left(q_{\max }\right) / D_{0}$ as well as $\gamma$ become $>1$ though the value of $\gamma$ stays close to $1(1.1<\gamma<1.4)$ indicating that though the contribution from the ballistic process is increasing, there is still a considerable contribution from the diffusive process leading towards de Gennes narrowing. Similar behavior has also been observed for the diagonal direction. However, the value of $D_{\text {eff }}\left(q_{\max }\right) / D_{0}$ and $\gamma$ is larger along the diagonal direction than along the parallel one.

In these discussions we have not considered any contributions from rotational diffusion, which in principle could also be detected by XPCS for anisotropic particles [47]. The scattering anisotropy of the cubelike particles used is not large enough to result in a significant depolarization ratio and thus measurable amplitudes for a contribution from rotational motion to the intensity autocorrelation function for individual particles. While this would be different for the large chains formed, their rotational motion is effectively suppressed due to the sedimentation-induced high concentration at the bottom of the capillary where we have performed our measurements, which can also be confirmed by optical microscopy. As a result we have not considered any effect on the correlation functions from the rotational motion of either the single particles or the chains.

We can therefore try to rationalize our observations by considering a superposition of diffusive and stress-induced ballistic processes. From the variation of the effective diffusion coefficients and the magnitude of $\gamma$, we observe that depending upon the direction of the scattering vector the relative contributions from these two processes change. For example, in the vertical direction, we do not observe the de Gennes narrowing, and the value of $\gamma$ is comparatively high. This indicates dominating contributions from ballistic processes. On the other hand, along the field direction not only do we observe the de Gennes narrowing, but also the value of $\gamma$ is close to 1 at zero field, which indicates that the contribution from the diffusive process is higher along this direction. A variation of the $q$ direction and the field strength thus allows us to correspondingly highlight the different processes.

\section{CONCLUSION}

Using ultrasmall-angle $\mathrm{x}$-ray photon correlation spectroscopy, we have studied the collective dynamics of colloidal cubes that are anisotropic in shape. Since the particles are made up of hematite cores, we were able to introduce anisotropy in their interaction potential with the help of an external magnetic field. We found that along with all measured azimuthal directions, $0^{\circ}, 45^{\circ}, 90^{\circ}$ with respect to the external field, the intermediate scattering function exhibits a 
compressed exponential decay at higher field strength, with the compressing exponent varying as a function of the direction and magnitude of $\vec{q}$ and the field strength. In addition, at $0^{\circ}$ and $45^{\circ}$ we observed structural correlation peaks along with a mild de Gennes narrowing in the effective diffusion coefficients at all magnetic fields. Our results qualitatively match with the model described in Ref. [40] where the authors attribute the discontinuity in the relaxation process to intermittent yet sudden rearrangement events.

In the case of magnetic colloids studied in this paper, the observed complex $\vec{q}$ and field strength dependence of the measured autocorrelation function on the probed characteristic length scales results from the rearrangements of the colloidal particles in the chains due to a combination of thermal and ballistic motion. The competition between the external magnetic and gravitational fields on the field-induced alignment of the dipolar chains gives rise to a heterogeneous local stress field which results in the ballistic motion of the particles. The comparisons of $D_{\text {eff }}\left(q_{\max }\right) / D_{0}$ and $\gamma$ along different azimuthal directions indicate that we have observed a weighted average of the two aforementioned processes for which the weighting factor depends on the azimuthal direction. In the perpendic- ular direction, ballistic motion is dominant, whereas in the parallel direction diffusive motion is dominant, while a mix of the two is found in the diagonal direction. The use of a fast two-dimensional detector in XPCS then allows us to probe the various mechanisms responsible for density fluctuations in the system through a variation of the magnitude and direction of $\vec{q}$ and field strength, which in turn affect the weight of their contributions to the measured intensity fluctuations. The success of our experiment paves a way towards exploring the anisotropic dynamics of colloidal suspensions and soft gels and solids at very low scattering vectors, which is important for investigations of the collective dynamics of self-assembled structures on mesoscopic length scales.

\section{ACKNOWLEDGMENTS}

C. Hetherington is acknowledged for TEM and T. Narayanan is acknowledged for the help during XPCS measurements. Financial support from the European $\mathrm{Re}$ search Council (Grant No. ERC-339678-COMPASS) and the Knut and Alice Wallenberg Foundation (Grant No. KAW 2014.0052), is gratefully acknowledged.
[1] P. J. Krommenhoek and J. B. Tracy, Magnetic field-directed self-assembly of magnetic nanoparticle chains in bulk polymers, Part. Part. Syst. Charact. 30, 759 (2013).

[2] J. Ge, Y. Hu, and Y. Yin, Highly tunable superparamagnetic colloidal photonic crystals, Angew. Chem. 119, 7572 (2007).

[3] L. He, Y. Hu, H. Kim, J. Ge, S. Kwon, and Y. Yin, Magnetic assembly of nonmagnetic particles into photonic crystal structures, Nano Lett. 10, 4708 (2010).

[4] V. Malik, A. V. Petukhov, L. He, Y. Yin, and M. Schmidt, Colloidal crystallization and structural changes in suspensions of silica/magnetite core-shell nanoparticles, Langmuir 28, 14777 (2012).

[5] A. Pal, V. Malik, L. He, B. H. Erné, Y. Yin, W. K. Kegel, and A. V. Petukhov, Tuning the colloidal crystal structure of magnetic particles by external field, Angew. Chem., Int. Ed. 54, 1803 (2015).

[6] J.-M. Meijer, D. V. Byelov, L. Rossi, A. Snigirev, I. Snigireva, A. P. Philipse, and A. V. Petukhov, Self-assembly of colloidal hematite cubes: A microradian x-ray diffraction exploration of sedimentary crystals, Soft Matter 9, 10729 (2013).

[7] E. van den Pol, A. V. Petukhov, D. V. Byelov, D. M. ThiesWeesie, A. Snigirev, I. Snigireva, and G. J. Vroege, Behavior of the smectic $A$ phase of colloidal goethite in a magnetic field, Soft Matter 6, 4895 (2010).

[8] D. V. Byelov, J.-M. Meijer, I. Snigireva, A. Snigirev, L. Rossi, E. van den Pol, A. Kuijk, A. Philipse, A. Imhof, A. van Blaaderen et al., In situ hard x-ray microscopy of self-assembly in colloidal suspensions, RSC Adv. 3, 15670 (2013).

[9] V. Malik, A. Pal, O. Pravaz, J. J. Crassous, S. Granville, B. Grobety, A. M. Hirt, H. Dietsch, and P. Schurtenberger, Hybrid magnetic iron oxide nanoparticles with tunable field-directed self-assembly, Nanoscale 9, 14405 (2017).

[10] I. Martchenko, J. J. Crassous, A. M. Mihut, E. Bialik, A. M. Hirt, C. Rufier, A. Menzel, H. Dietsch, P. Linse, and P.
Schurtenberger, Anisotropic magnetic particles in a magnetic field, Soft Matter 12, 8755 (2016).

[11] J. J. Crassous, A. M. Mihut, E. Wernersson, P. Pfleiderer, J. Vermant, P. Linse, and P. Schurtenberger, Field-induced assembly of colloidal ellipsoids into well-defined microtubules, Nat. Commun. 5, 1 (2014).

[12] D. J. Kraft, R. Ni, F. Smallenburg, M. Hermes, K. Yoon, D. A. Weitz, A. van Blaaderen, J. Groenewold, M. Dijkstra, and W. K. Kegel, Surface roughness directed self-assembly of patchy particles into colloidal micelles, Proc. Natl. Acad. Sci. USA 109, 10787 (2012)

[13] M. Grzelczak, L. M. Liz-Marzán, and R. Klajn, Stimuliresponsive self-assembly of nanoparticles, Chem. Soc. Rev. 48, 1342 (2019).

[14] B. Bharti and O. D. Velev, Assembly of reconfigurable colloidal structures by multidirectional field-induced interactions, Langmuir 31, 7897 (2015).

[15] A. Pal, V. A. Martinez, T. H. Ito, J. Arlt, J. J. Crassous, W. C. Poon, and P. Schurtenberger, Anisotropic dynamics and kinetic arrest of dense colloidal ellipsoids in the presence of an external field studied by differential dynamic microscopy, Sci. Adv. 6, eaaw9733 (2020).

[16] A. Pal, T. Zinn, M. A. Kamal, T. Narayanan, and P. Schurtenberger, Anomalous dynamics of magnetic anisotropic colloids studied by XPCS, Small 14, 1802233 (2018).

[17] A. J. Banchio, J. Gapinski, A. Patkowski, W. Häußler, A. Fluerasu, S. Sacanna, P. Holmqvist, G. Meier, M. P. Lettinga, and G. Nägele, Many-Body Hydrodynamic Interactions in Charge-Stabilized Suspensions, Phys. Rev. Lett. 96, 138303 (2006).

[18] A. Robert, E. Wandersman, E. Dubois, V. Dupuis, and R. Perzynski, Glassy dynamics and aging in a dense ferrofluid, Europhys. Lett. 75, 764 (2006). 
[19] K. L. Brinker, S. G. Mochrie, and W. R. Burghardt, Equilibrium dynamics of a polymer bicontinuous microemulsion, Macromolecules 40, 5150 (2007).

[20] A. Papagiannopoulos, T. Waigh, A. Fluerasu, C. Fernyhough, and A. Madsen, Microrheology of polymeric solutions using Xray photon correlation spectroscopy, J. Phys.: Condens. Matter 17, L279 (2005).

[21] V. Trappe, E. Pitard, L. Ramos, A. Robert, H. Bissig, and L. Cipelletti, Investigation of $q$-dependent dynamical heterogeneity in a colloidal gel by x-ray photon correlation spectroscopy, Phys. Rev. E 76, 051404 (2007).

[22] A. Fluerasu, A. Moussaïd, A. Madsen, and A. Schofield, Slow dynamics and aging in colloidal gels studied by $\mathrm{x}$-ray photon correlation spectroscopy, Phys. Rev. E 76, 010401(R) (2007).

[23] C. Caronna, Y. Chushkin, A. Madsen, and A. Cupane, Dynamics of Nanoparticles in a Supercooled Liquid, Phys. Rev. Lett. 100, 055702 (2008)

[24] H. Guo, G. Bourret, M. K. Corbierre, S. Rucareanu, R. B. Lennox, K. Laaziri, L. Piche, M. Sutton, J. L. Harden, and R. L. Leheny, Nanoparticle Motion within Glassy Polymer Melts, Phys. Rev. Lett. 102, 075702 (2009).

[25] T. Sun, B. Yao, A. P. Warren, K. Barmak, M. F. Toney, R. E. Peale, and K. R. Coffey, Dominant role of grain boundary scattering in the resistivity of nanometric Cu films, Phys. Rev. B 79, 041402(R) (2009).

[26] E. Wandersman, Y. Chushkin, E. Dubois, V. Dupuis, A. Robert, and R. Perzynski, Field induced anisotropic cooperativity in a magnetic colloidal glass, Soft Matter 11, 7165 (2015).

[27] E. Wandersman, E. Dubois, V. Dupuis, A. Duri, A. Robert, and R. Perzynski, Heterogeneous dynamics and ageing in a dense ferro-glass, J. Phys.: Condens. Matter 20, 204124 (2008).

[28] E. Wandersman, A. Duri, A. Robert, E. Dubois, V. Dupuis, and R. Perzynski, Probing heterogeneous dynamics of a repulsive colloidal glass by time resolved $\mathrm{x}$-ray correlation spectroscopy, J. Phys.: Condens. Matter 20, 155104 (2008).

[29] R. Steinmann, Y. Chushkin, C. Caronna, J. Chavanne, and A. Madsen, A small-angle scattering chamber for x-ray photon correlation spectroscopy at low temperatures, Rev. Sci. Instrum. 82, 025109 (2011).

[30] J. Lal, D. Abernathy, L. Auvray, O. Diat, and G. Grübel, Dynamics and correlations in magnetic colloidal systems studied by x-ray photon correlation spectroscopy, Eur. Phys. J. E 4, 263 (2001).

[31] J. Wagner, C. Märkert, B. Fischer, and L. Müller, Direction Dependent Diffusion of Aligned Magnetic Rods by Means of X-Ray Photon Correlation Spectroscopy, Phys. Rev. Lett. 110, 048301 (2013).

[32] A. Schavkan, Dynamics of colloidal systems of magnetic nanoparticles under influence of magnetic fields investigated by XPCS, DESY thesis, Deutsches Elektronen-Synchrotron DESY, 2017.
[33] T. Sugimoto and K. Sakata, Preparation of monodisperse pseudocubic $\alpha-\mathrm{Fe}_{2} \mathrm{O}_{3}$ particles from condensed ferric hydroxide gel, J. Colloid Interface Sci. 152, 587 (1992).

[34] C. Graf, D. L. Vossen, A. Imhof, and A. van Blaaderen, A general method to coat colloidal particles with silica, Langmuir 19, 6693 (2003).

[35] F. Lehmkühler, P. Kwaśniewski, W. Roseker, B. Fischer, M. A. Schroer, K. Tono, T. Katayama, M. Sprung, M. Sikorski, S. Song et al., Sequential single shot x-ray photon correlation spectroscopy at the SACLA free electron laser, Sci. Rep. 5, 17193 (2015).

[36] L. Rossi, J. G. Donaldson, J.-M. Meijer, A. V. Petukhov, D. Kleckner, S. S. Kantorovich, W. T. Irvine, A. P. Philipse, and S. Sacanna, Self-organization in dipolar cube fluids constrained by competing anisotropies, Soft Matter 14, 1080 (2018).

[37] J. Möller, Y. Chushkin, S. Prevost, and T. Narayanan, Multispeckle $\mathrm{x}$-ray photon correlation spectroscopy in the ultrasmall-angle x-ray scattering range, J. Synchrotron Radiat. 23, 929 (2016).

[38] T. Zinn, A. Homs, L. Sharpnack, G. Tinti, E. Fröjdh, P.A. Douissard, M. Kocsis, J. Möller, Y. Chushkin, and T. Narayanan, Ultra-small-angle $\mathrm{x}$-ray photon correlation spectroscopy using the Eiger detector, J. Synchrotron Radiat. 25 1753 (2018).

[39] C. Lindsey and G. Patterson, Detailed comparison of the Williams-Watts and Cole-Davidson functions, J. Chem. Phys. 73, 3348 (1980).

[40] A. Duri and L. Cipelletti, Length scale dependence of dynamical heterogeneity in a colloidal fractal gel, Europhys. Lett. $\mathbf{7 6}$, 972 (2006).

[41] L. Cipelletti, L. Ramos, S. Manley, E. Pitard, D. A. Weitz, E. E. Pashkovski, and M. Johansson, Universal non-diffusive slow dynamics in aging soft matter, Faraday Discuss. 123, 237 (2003).

[42] A. J. Banchio and G. Nägele, Short-time transport properties in dense suspensions: From neutral to charge-stabilized colloidal spheres, J. Chem. Phys. 128, 104903 (2008).

[43] P. G. De Gennes, Liquid dynamics and inelastic scattering of neutrons, Physica 25, 825 (1959).

[44] K. Nygård, J. Buitenhuis, M. Kagias, K. Jefimovs, F. Zontone, and Y. Chushkin, Anisotropic de Gennes Narrowing in Confined Fluids, Phys. Rev. Lett. 116, 167801 (2016).

[45] P. Falus, M. A. Borthwick, S. Narayanan, A. R. Sandy, and S. G. J. Mochrie, Crossover from Stretched to Compressed Exponential Relaxations in a Polymer-Based Sponge Phase, Phys. Rev. Lett. 97, 066102 (2006).

[46] J.-P. Bouchaud and E. Pitard, Anomalous dynamical light scattering in soft glassy gels, Eur. Phys. J. E 6, 231 (2001).

[47] P. Holmqvist, V. Meester, F. Westermeier, and D. Kleshchanok, Rotational diffusion in concentrated platelet systems measured with X-ray photon correlation spectroscopy, J. Chem. Phys. 139, 084905 (2013). 